\title{
Melatonin changes the electrical spontaneous activity of hippocampal rat neurons at different ages
}

\author{
Daniel Perez-Torres, Cruz Reyes-Vázquez, Mireya Velázquez-Paniagua, Bertha Prieto-Gómez*
}

Facultad de Medicina, Universidad Nacional Autónoma de México, México D.F., México;

*Corresponding Author: prieto@unam.mx, ketevayabonito@gmail.com, crv@unam.mx, yetlanetzi@unam.mx

Received 30 October 2013; revised 30 November 2013; accepted 7 December 2013

Copyright (c) 2014 D. Perez-Torres et al. This is an open access article distributed under the Creative Commons Attribution License, which permits unrestricted use, distribution, and reproduction in any medium, provided the original work is properly cited. In accordance of the Creative Commons Attribution License all Copyrights (C) 2014 are reserved for SCIRP and the owner of the intellectual property D. Perez-Torres et al. All Copyright (C) 2014 are guarded by law and by SCIRP as a guardian.

\section{ABSTRACT}

Melatonin, the pineal indole is characterized by being a compound that crosses all cell membranes and which has been attributed to several mechanisms of action. Among these it is the ability to reduce free radicals, thereby reducing the potential aging and cell death. Studies in different age Wistar rats have shown that chronic application of melatonin, in the hippocampus, reduces the concentration of free radicals and keeps its architecture. This study showed that melatonin increases the firing rate and favors the presence of bursting activity in animals of different ages. It is suggested that melatonin conserved hippocampal cells in good anatomical and physiological condition probably as a result of the elimination of free radicals.

\section{KEYWORDS}

Melatonin; Unitary Neuronal Activity; Hippocampus; Rat; Age

\section{INTRODUCTION}

Aging is a progressive and complex procedure during life that includes gradual cellular loss, endocrine and metabolic deficits, decreasing defense mechanisms and functional losses [1], which contributes to health deterioration and ultimately to death with the passage of time. During this process there is a general decline in nervous system functions due to structural, molecular, biochemical and physiological changes [2]. The brain's volume and weight decay with age at a rate of around 5\% per decade after age 40 with a decline possibly increasing with age particularly over age 70 [3]. At the beginning of the decade of 2000 it was suggested that the shrinking of the grey matter could be the result of neuronal cell death [4]. However, numerous observations indicate that there is a widespread preservation of anatomy and function in the aging brain; in particular, in the hippocampus the changes that occur are relatively specific for different subregions of this area [5]. Thus, the hippocampus is a particular cerebral structure associated with the cognitive impairment in the aging [6,7]. Several data suggest changes in the morphology of the hippocampus during aging [8,9], however several anatomical studies associated with aging have observed no major loss of hippocampal cells, but it seems that there are changes in the functional dynamics of the hippocampus due to alterations in the connectivity between cells [5]. So, CA1 was observed that there are no loss of synaptic contacts with both the Schaffer collateral fibers as the axonal projections with cells of the entorhinal cortex but it has been reported that in aged animals the hippocampal synapses are no longer functional or acquire a silent state [10]. Therefore, physiological changes in the aged brain could be expressed as the decline in the neuronal tissue physicological activity, for example, changes in spontaneous multiple-unit activity can be considered as a measure of neural impairment and thus as an important parameter of electrophysiological ageing and decrement in neurological functions [2].

Furthermore, in aging, free radicals have an important role because these molecules are highly reactive and they are able to chemically modify other molecules. In normal aging there is an increase of free radicals, mainly reactive oxygen species (ROS). Although these physiological ROS acting as intracellular signaling molecules also can oxidize DNA, lipids and proteins provoking cell damage and apoptosis, leading to the structural and functional tissue decline [1]. The ROS production leads to an imbalance in the redox status damaging the mitochondrial 
electron transport chain and augmenting the free radical generation, leading the mitochondrial ROS to flux to cytoplasm where they induce calcium release from the endoplasmic reticulum, resulting in mitochondrial calcium uptake generating a mitochondrial dysfunction associated with the loss of calcium homeostasis and enhancement of cellular oxidative stress. This mitochondrial dysfunction is considered as the driving force for the ageing process [11].

Melatonin (a pineal's indol), due to its capacity to cross all cellular membranes (can be especially concentrated in the nucleus and mitochondria) [12], has been considered among the very best free radical scavengers [13]. Melatonin can act as a direct antioxidant, acting as a free radical scavenger removing free radicals or through the synthesis of antioxidant metabolites like $\mathrm{N}$-acetyl- $\mathrm{N}$ formyl-5-methoxy-kynuramine and $N$-acetyl-methoxykynuramine [14], and as an indirect antioxidant agent through second messengers, including cyclic nucleotides, diacylglycerol, arachidonic acid, intracellular calcium concentration and several transcription factors [15]. It also elicits the increment in the expression and activity of some antioxidant enzymes, such as Superoxide dismutase, Catalase, Glutathione peroxidase and Glutathione reductase [14].

Some radicals, as the ROS, which are reduced by melatonin, in their moment can injure the cells and possibly lead them to the death $[16,17]$. As melatonin decreases with aging and therefore its antioxidant effects, also several neurodegenerative diseases have been associated with its plasmatic decrement and with the increase of the amount of free radicals producing oxidative stress which would lead to the neuronal deterioration in some of these diseases [17-19].

It is important to note that in the hippocampus there are melatonin receptors $[20,21]$ which show that this hormone can bind to the cells of this region and play an important role in the aging [22,23].

In recent years our laboratory described, in Wistar rats, that the hippocampus has an increase in the concentration of free radicals which is parallel to the increasing age of the rats. The chronic application of melatonin significantly decreased the concentration of these radicals in the rat hippocampus at different ages, measured by electronic paramagnetic resonance [24]. It suggests that melatonin can protect this cerebral area from the oxidation. These data are in accord with some investigations in vitro which support the idea that in the central nervous system melatonin appears to play a protective role in neuronal processes $[25,26]$.

Whether reducing free radicals there is a decrease in cellular oxidation then diminish cell death. So, it was proposed that the architecture of the hippocampus would remain in top condition in animals treated with melatonin that in animals did not treat with this indole. Thus in a second study it was showed that the mentioned hormone elicited a higher expression of the MAP 2 in both CA1 and CA3 in the hippocampus at different ages. However, we could not determine whether the effect was due to the better preserved neurite or if there were new neuronal structures [27]. This paper argues that if melatonin reduces free radicals and keeps in "good anatomical condition" to the hippocampus then it must also maintain the electrophysiological activity of its cells properly which is raised to analyze the effect of the hormone on the unitary, spontaneous electrical activity of the hippocampus at different ages.

\section{METHOD}

Seventy six Wistar male rats of two months of age (180 - 200 g body weight), remained under, laboratory conditions with a temperature of $23^{\circ} \mathrm{C} \pm 2^{\circ} \mathrm{C}$, and a cycle light-dark 12/12 hours, water and food ad libitum. The animals were divided in 2 groups, one received melatonin in tap water $(0.5 \mathrm{mg} / \mathrm{rat} / \mathrm{day})$ and the control group received polyethylene glycol (1\%, melatonin vehicle) every day. The treatment was performed by two, four, six and eight months (middle-aged rats).

Unitary electrophysiological recordings were made in urethane-anesthetized animals, $(1.5 \mathrm{mg} / \mathrm{Kg})$. On the recording day, the animal was placed in a stereotaxic apparatus and following the coordinates of the of Pellegrino's atlas [28], a chloridized silver electrode into a glass pipette filled with $\mathrm{NaCl} 4 \mathrm{M}$ and a dye was introduced to the CA1 hippocampal area. Once that spontaneous unitary activity in the hippocampus was found, this was recorded by at least $15 \mathrm{~min}$ and when it became stabilized was recorded by at least 60 minutes more. Several cells were recorded in each rat. Once finished the recording, a cathodic current was injected for depositing the dye and to mark the registered area. The animal was sacrificed with deep anesthesia and the brain was extracted for its later histological analysis. With the collected data, by means of a discrimination window [Winston Electronics $\mathrm{CO}$ ], frequency histograms were made to determine firing pattern of each cell. The recording procedures were made at 2, 4, 6 and 8 months of treatment.

\section{STATISTICAL ANALYSIS}

To determine the frequency of spontaneous activity, the number of spikes in time was obtained (number of spikes/60 seg). Frequency histograms were developed for each cell (v10.0 Sigma Plot). And the average frequency was obtained from each recorded cell en each age (Table 1).

Due to the non-standard distribution of data from 
Table 1. It shows the average activity in both groups; the control and the melatonin-treated animals, at all ages recorded. There are differences between the two groups, but only there are significant differences until the 8 month of treatment.

\begin{tabular}{ccccc}
\hline GROUP & 2 months & 4 months & 6 months & 8 months \\
\hline Control & $337.14 \pm 85.24$ & $278.55 \pm 67.63$ & $263.45 \pm 65.64$ & $258.42 \pm 57.01$ \\
Melatonin & $415.33 \pm 122.45$ & $409.93 \pm 92.20$ & $466.00 \pm 131.14$ & $443.10 \pm 75.37^{*}$ \\
\hline
\end{tabular}

*Indicates a significant difference in the electrophysiological activity of rats treated with melatonin at 8 months of treatment compared to control.

electrophysiological recordings in area CA1 of the hippocampus, we performed data normalization with reference to the maximum frequency value obtained for each group making it equal to 1 , the maximum value was taken as reference to normalize the distribution, and have better statistical treatment of the data.

The mean of normalized data was taken for each group, the control and that of melatonin. Statistical comparisons between both groups were carried out by using Student's t-test (significance level to $\mathrm{p}<0.05$ ).

\section{RESULTS}

Spontaneous action potentials in hippocampal cells from 76 rats were recorded. The number of cells was different for each age. We report here only cells that were recorded for at least 60 minutes after its stability.

In the CA1 area of control rats were obtained discharge patterns with unique spikes (tonic) while in the same area, the melatonin-treated rats, this pattern, showed a shift in the timing of spontaneous activity with a phasic pattern trend (the phasic distribution is characterized by aggregates of action potentials in a period of time with alternated quiescent states of neuronal activity) generated by trains of activity and unitary activity. There was a decrease in activity frequency starting at four months of age (Table 2).

At two months of treatment the rats dealt with melatonin exhibited a bursting activity with a frequency higher than in the control rats, which exhibited a tonic activity. The dispersion was higher in the melatonin rats than that found in control rats. There were no significant differences (Figure 1).

After four months of treatment, control rats shown a discharge pattern with unique spikes (tonic pattern) and the frequency is lesser than that observed in rats treated with melatonin. In the last group in clear the phasic pattern exhibited by the experimental rats. There were no differences (Figure 2).

At six months the control rats exhibited a tonic pattern activity while the cells from melatonin treated rats shown a phasic and a tonic pattern with a frequency higher than that displayed by the control rats. There were no differences (Figure 3).

At eight months of treatment the cells from control rats showed, principally, discharge patterns with unique spikes; however some cells exhibited a phasic pattern,

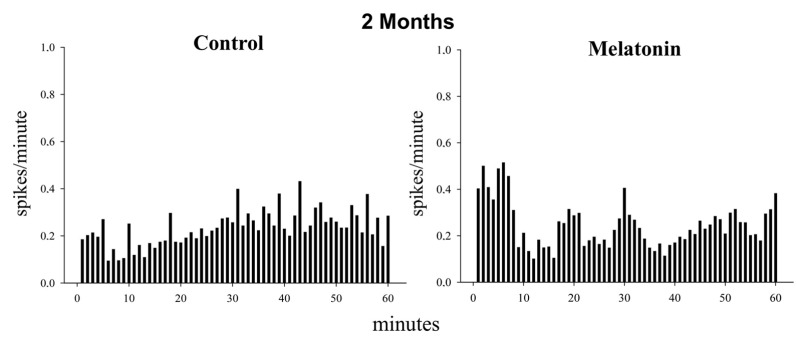

Figure 1. The figure shows histograms of frequency (spikes/ minute) in two recorded neurons (60 minutes) in hippocampal CA1. In the control cell is observed a tonic firing pattern while in the cell treated with melatonin there is a phasic discharge pattern. The frequencies were similar. Rats were 2 months of treatment. The data shown are normalized.

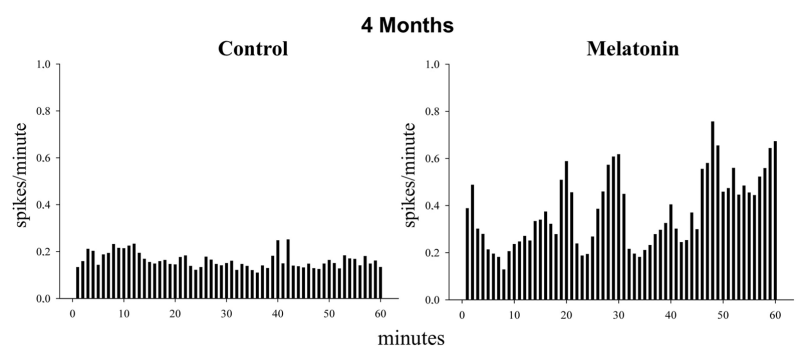

Figure 2. The figure shows histograms of frequency (spikes/ minute) in two recorded neurons (60 minutes) in hippocampal CA1. In the control cell is observed a tonic firing pattern while in the cell treated with melatonin there is a phasic discharge pattern. Discharge frequency was about twice that exhibited by control cells. The rats were 4 months of treatment. The data shown are normalized.

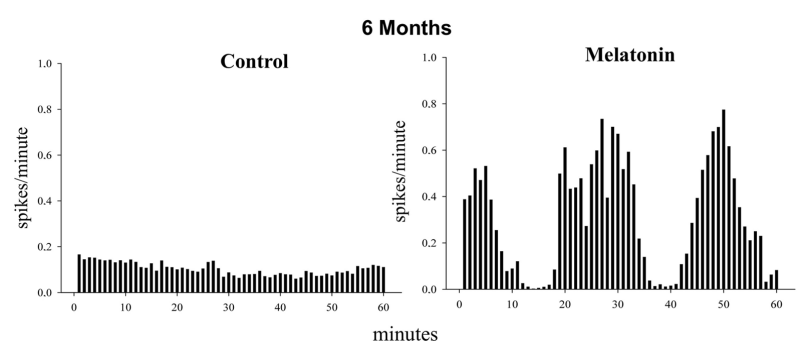

Figure 3. The figure shows histograms of frequency (spikes/ minute) in two recorded neurons (60 minutes) in hippocampal CA1. In the control cell observed tonic firing pattern while in the cell treated with melatonin there is a phasic discharge pattern. It is noticeable that the activity in bursts is greater than the tonic in this cell and is almost twice than the control. The rats were 6 months of treatment. The histogram data shown are normalized. 
Table 2. The table shows the percentage of the patterns of neuronal activity per group ( $\mathrm{C}=$ control and $\mathrm{M}=$ melatonin) and for the time of treatment (months). The percentage of the characteristic neuronal firing pattern of spontaneous activity in CA1 recorded in each group showed that in melatonin groups $(\mathrm{M})$ at each month there was mainly, phasic patterns characterized by the mixing of unitary and bursting activity, whereas in control groups the pattern activity was mainly tonic .For each treatment $\mathrm{n}=30$. The pattern of neuronal activity in the rest of neurons from each group was not classified because the apparent transition of one to other firing type or because there was a pattern of neural activity well established.

\begin{tabular}{cccccccccc}
\hline & \multicolumn{2}{c}{2 months } & \multicolumn{2}{c}{ 4 months } & \multicolumn{2}{c}{6 months } & & 8 months \\
\hline $\begin{array}{c}\text { Number of } \\
\text { neurons }\end{array}$ & C & M & C & M & C & M & C & M \\
$\begin{array}{c}\text { \% neuronal } \\
\text { firing pattern }\end{array}$ & Tonic 90\% & Phasic 70\% & Tonic 80\% & Phasic 83.3\% & Tonic 76.6\% & Phasic 73.3\% & Tonic 56.6\% & Phasic 76.6\% \\
\hline
\end{tabular}

while all the cells from rats treated with melatonin exhibit bursting activity. There were differences to $\mathrm{p}<0.05$. (Figure 4)

The Figure 5 displays the average firing frequency normalized in both groups. The control rats exhibited a similar activity at 2, 4, and 6 months while at 8 months this activity decreased even more. Melatonin treated cells shown an activity which was increasing with the time and always was higher than the control activity

\section{DISCUSSION}

The firing pattern from hippocampal area in rats, chronically treated with melatonin at different age was characterized, and it was determined whether hippocampal cells with "greater structural stability" had a "stable" electrical activity. The animals, showed a significant increase in the hippocampal unitary activity. The cell from control rats showed mainly discharges of simple spikes, while cells of rats treated with melatonin showed predominantly high frequency bursting activity.

In our work, melatonin treated rats, they were middle age animals (10 months old), exhibited an increase in the spontaneous activity of hippocampus, which can be in accordance with the findings of Musshoff et al. [20], in vitro, shown the enhance of spontaneous firing rate of neurons in CA1 region after melatonin application. One possibility of this increase could be that melatonin application elicits a reduction of free radicals in this hippocampal area as it has been showed [24] and so the cells have less oxidative damage and therefore the melatonin recorded cells are in a better anatomical and physiological condition than the control.

A number of studies have postulated that aging synaptic function is the result from the alteration of intracellular calcium levels which can result in changes in the transmission of information through the nervous system [29]. Melatonin modulates intracellular levels of calcium by receptors, found primarily in dentate gyrus, CA3, CA1, and subiculum, and by regulating the high-voltageactivated calcium channel, and it opens $\mathrm{Ca}^{2+}$ intracellular stores. In addition, within the cell, melatonin interacts

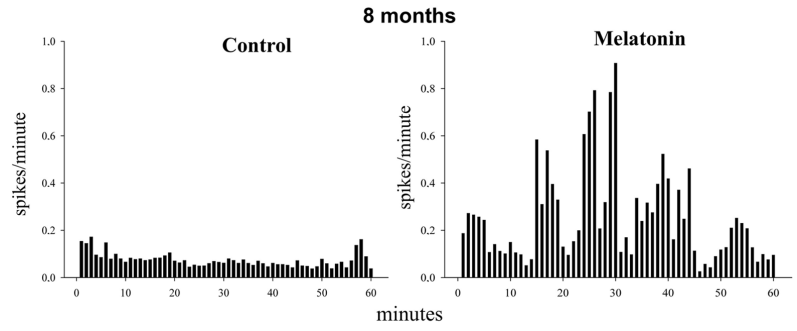

Figure 4. The figure shows histograms of frequency (spikes/ minute) in two recorded neurons (60 minutes) in hippocampal CA1. In the control cell observed tonic firing pattern, whereas in the cell treated with melatonin there is a phasic discharge pattern. It is noticeable that the activity in bursts is greater than the tonic in this cell. Clearly the control cell showed a very low frequency compared to the experimental cell, discharge frequency shown by the two groups were statistically different at $\mathrm{p}$ $<0.05$. The rats were 8 months of treatment. In the histogram, the data shown are normalized.

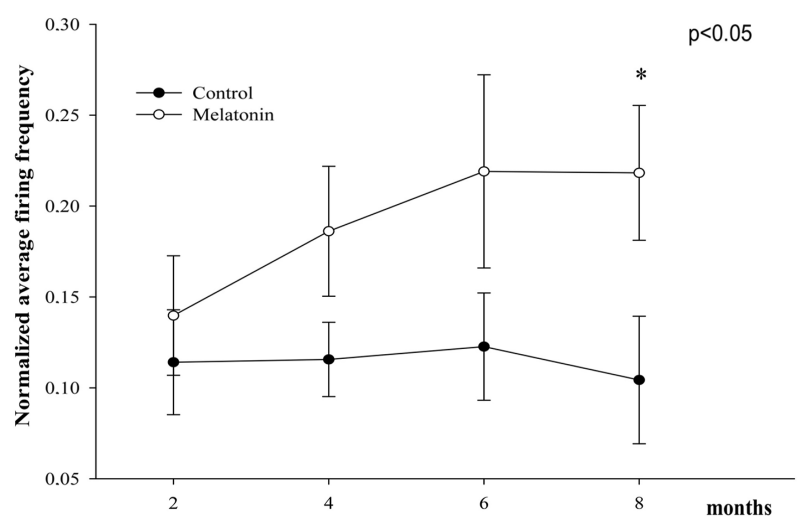

Figure 5. The figure shows the normalized average firing frequency in control and experimental rats at 4 ages recorded. It is observed that the frequency in control rats was very similar in the first three ages while at 8 months of treatment there was a decrease of this. Melatonin-treated rats from two months of treatment show a higher activity than the control rats. However, the dispersion in such activity is also higher than in the controls, finding significant differences only at 8 months of treatment.

with several proteins, calmodulin, G-protein, protein Kinase $\alpha$ and adenylyl cyclasa which play an important role in the calcium signaling [30]. In this way several in 
vitro evoked potentials studies had shown the multifaceted effects of melatonin in synaptic activity such as reduction in the amplitude of synaptically evoked responses, no effect on synaptic transmission, a biphasic effect with a first depression followed by a recovery/amplification phase [15], decrements in the current amplitude of GABAA receptors [31], or the block effects of melatonin on synaptic transmission and LTP processes in the CA1 hippocampal neurons [15,20,30]. However, on the contrary to the accepted view of the inhibitory action of melatonin on neural activity, neurons in CA1 region shown an increase of spontaneous firing rate after chronic melatonin application as it has been mentioned in vitro experiments [30].

The continuous use of melatonin, as is the case in this study, could produce changes in the calcium dynamic and elicit changes in the rate and pattern of neuronal firing. Our data show that in cells from melatonin treated rats, increased spontaneous activity and even firing pattern change, which could be related with changes in the calcium dynamics.

In this way, the fact that the levels of this indolemine in different tissues are inversely related to the oxidative damage [1,12] (indicates that the use of melatonin as an antioxidant factor is possible as experimental treatment in dementia senile and neurodegenerative diseases [14, 32]).

\section{CONCLUSIONS}

The chronic application of melatonin in rat inducing neuronal frequencies of firing in the hippocampus is greater than the one observed in control rats.

The increase elicited by melatonin in the firing rate could result by reducing the free radicals, maintaining the anatomic architecture and probably by modifying the signalization mechanisms, perhaps the calcium dynamics, however this last assertion must be studied in the complete animal.

It is concluded that in aged animals, melatonin application can protect the electrophysiological activity which could preserve the mechanisms necessary for memory formation and reduce the alterations in this parameter observed in old patients.

\section{REFERENCES}

[1] López, L.C., Escames, G., López, A. and García, J.A. Doerrier, C. and Acuña-Castroviejo, D. (2010) Melatonin, neurogenesis, and aging brain. The Open Neuroendocrinology Journal, 3, 121-133.

[2] Rameswhar, S. and Sharma, D. (2005) Electrophysiology ageing of the brain and pharmacology of ageing. In: Thakur, M.K. and Prasad, S., Eds., Molecular and Cellular Neurobiology, Narosa Publishing House Pvt. Ltd.,
New Delhi, 135-148.

[3] Peters, R. (2006) Ageing and the brain. Postgraduate Medical Journal, 82, 84-88. http://dx.doi.org/10.1136/pgmj.2005.036665

[4] Anderson, B.H. (2002) Ageing of the brain. Mechanisms of Ageing and Development, 123, 811-817. http://dx.doi.org/10.1016/S0047-6374(01)00426-2

[5] Rosenzweig, E.S. and Barnes, C.A. (2003) Impact of aging on hippocampal function: plasticity, network dynamic, and cognition. Progress in Neurobiology, 69, 143179. http://dx.doi.org/10.1016/S0301-0082(02)00126-0

[6] Gallagher, M. (1997) The use of animal models to study the effects of aging on cognition. Annual Review of Psychology, 48, 339-370.

http://dx.doi.org/10.1146/annurev.psych.48.1.339

[7] Small, S.A., Schobel, S.A., Buxton, R.B., Witter, M.P. and Barnes, C.A. (2011) A pathophysiological framework of hippocampal dysfunction in ageing and disease. Nature Reviews Neuroscience, 12, 585-601. http://dx.doi.org/10.1038/nrn3085

[8] Jessberger, S. and Gage, F.H. (2008) Stem-cell-associated structural and functional plasticity in the aging hippocampus. Psychology and Aging, 23, 684-691. http://dx.doi.org/10.1037/a0014188

[9] Dickstein, D.L. Weaver C.M., Luebke, J.I. and Hof, P.R. (2013) Dendritic spine changes associated with normal aging. Neuroscience, 22, 21-32.

http://dx.doi.org/10.1016/j.neuroscience.2012.09.077

[10] Burke, S.N. and Barnes, C.A. (2010) Senescent synapses and hippocampal circuit dynamics. Trends in Neurosciences, 33, 153-161.

http://dx.doi.org/10.1016/j.tins.2009.12.003

[11] Srinivasan, V., Pandi-Perumal, S.R., Cardinali, D.P., Poeggeler, B. and Hardeland, R. (2006) Melatonin in Alzheimer's disease and other neurodegenerative disorders. Behavioral and Brain Functions, 4, 2-15.

[12] Escames, G., López, A., García, J.A., García, L., AcuñaCastroviejo, D., García, J.J. and López, L.C. (2010) The role of mitochondria in brain aging and the effects of melatonin. Current Neuropharmacology, 8, 182-193. http://dx.doi.org/10.2174/157015910792246245

[13] Reiter, R.J., Melchiorri, D., Sewerynek, E., Poeggeler, B., Barlow-Walden, L., Chuang, J., Ortiz, G.G. and AcuñaCastroviejo, D. (1995) A review of the evidence supporting melatonin's role as an antioxidant. Journal of Pineal Research, 18, 1-11. http://dx.doi.org/10.1111/j.1600-079X.1995.tb00133.x

[14] Tomás-Zapico, C. and Coto-Montes, A. (2007) Melatonin as antioxidant under pathological processes. Recent $\mathrm{Pa}$ tents on Endocrine, Metabolic \& Immune Drug Discovery, 1, 63-82.

[15] Ozcan, M., Yilmaz, B. and Carpenter, D.O. (2006) Effects of melatonin on synaptic trasmission and long-term potentiation in two areas of mouse hippocampus. Brain Research, 1111, 90-94. http://dx.doi.org/10.1016/j.brainres.2006.06.117

[16] Chawla, S.L. and Rao, M.V. (2012) Protective effect of melatonin against fluorideinduced oxidative stress in the 
mouse ovary. Research Report Fluoride, 45, 125-132.

[17] Reiter R.J. and Benitez-King G. (2009) Melatonin reduces neuronal loss and cytoskeletal. Salud Mental, 32, 3-11.

[18] Srinivasan, V., Spence, D.W., Pandi-Perumal, S.R., Brown Gregory, M. and Cardinali Daniel, P. (2011) Melatonin in mitochondrial dysfunction and related disorders. International Journal of Alzheimer's Disease, 2011, Article ID: 326320.

[19] Gupta, Y.K., Gupta, M. and Kohli, K. (2003) Neuroprotective role of melatonin in oxidative stress vulnerable brain. Indian Journal of Physiology and Pharmacology, 47, 373-386.

[20] Musshoff, U., Riewenherm, D., Berger, E., Fauteck, J.D. and Speckmann, E.J. (2002) Melatonin receptors in rat hippocampues: Molecular and functional investigations. Hippocampus, 12, 165-173. http://dx.doi.org/10.1002/hipo.1105

[21] Dubocovich, M.L., Delagrange, P., Krause, D.N., Sugden, D., Cardinali, D.P. and Olcese, J. (2010) International union of basic and clinical pharmacology. LXXV. Nomenclature, classification, and pharmacology of G protein-coupled melatonin receptors. Pharmacological Reviews, 62, 343-380. http://dx.doi.org/10.1124/pr.110.002832

[22] Lee, C.H., Choi, J.H., Yoo, K.Y., Park, O.K., Hwang, I.K., You, S.G., Lee, B.Y., Kang, I.J. and Won, M.H. (2010) MT2 melatonin receptor immunoreactivity in neurons is very high in the aged hippocampal formation in gerbils. Cellular and Molecular Neurobiology, 30, 255-263. http://dx.doi.org/10.1007/s10571-009-9447-6

[23] Cavanaugh, J.E. and Witt-Enderby Paul, A. (2010) Mini-review: CNS melatonin receptors and signaling: Focus on aging-related diseases and future perspectives. The Open Neuroendocrinology Journal, 3, 96-104.

[24] Velázquez, P.M, Contreras, P.R., Gómez, V.V., Meneses, M.A. and Prieto, G.B. (2007) Melatonin's effects on free radical levels in rat hippocampus, measured by electronic paramagnetic resonance. Pharmacologyonline, 1, 170-
175.

[25] Reiter, R.J., Tan, D.X., Mayo, J.C, Sainz, R.M., Leon, J. and Czarnocki, Z. (2003) Melatonin as an antioxidant: biochemical mechanisms and pathophysiological implications in humans. Acta Biochimica Polonica, 50, 11291146.

[26] Benítez, K.G. (2006) Melatonin as a cytoskeletal modulator: Implications for cell physiology and disease. Journal of Pineal Research, 40, 1-9. http://dx.doi.org/10.1111/j.1600-079X.2005.00282.x

[27] Prieto, G.B., Velázquez, P.M., Olivos, C.L., Reyes, V.C., Jiménez, T.F., Reyes, ME., Mendoza, T.J. and Gutiérrez, O.G. (2008) Melatonin attenuates the decrement of dendritic protein MAP-2 immuno-staining in the hippocampal CA1 and CA3 fields of the aging male rat. Neuroscience Letters, 448, 56-61. http://dx.doi.org/10.1111/j.1600-079X.2005.00282.x

[28] Pellegrino, J.L., Pellegrino, S.A. and Cushman, J.A. (1986) A stereotaxic atlas of the rat brain. Plenum Press, New York, 4-122.

[29] Foster, T.C. (2007) Calcium homeostasis and modulation of synaptic plasticity in aged brain. Aging Cell, 6, 319325. http://dx.doi.org/10.1111/j.1474-9726.2007.00283.x

[30] Poloni, J.F., Feltes, B.C. and Bonatto, D. (2011) Melatonin as a central molecule connecting neural development and calcium signaling. Functional \& Integrative Genomics, 11, 383-388. http://dx.doi.org/10.1007/s10142-011-0221-8

[31] Wan, Q., Man, H.Y., Liu, F., Braunton, J., Niznik, H.B., Fun, P.S., Brown, G.M. and Wang, Y.T. (1999) Differential modulation of GABAA receptor function by Mel1a and Mel1b receptors. Nature Neuroscience, 2, 401-403.

[32] Luchetti, F., Canonico, B., Betti, M., Ancandeletti, M., Pilolli, F., Piroddi, M., Canesi, L., Papa, S. and Galli, F. (2010) Melatonin signaling and cell protection function. 24, 3603-3624. 